\title{
Change in left ventricular velocity time integral during Trendelenburg maneuver predicts fluid responsiveness in cardiac surgical patients in the operating room
}

\author{
Guo-Guang Ma ${ }^{1, \#}$, Li-Ying $\mathrm{Xu}^{3 \#}$, Jing-Chao Luo ${ }^{1 \#}$, Jun-Yi Hou ${ }^{1}$, Guang-Wei Hao ${ }^{1}$, Ying Su ${ }^{1}$, Kai Liu ${ }^{1}$, \\ Shen-Ji Yu ${ }^{1}$, Guo-Wei Tu' ${ }^{1}$, Zhe Luo ${ }^{1,4}$ \\ ${ }^{1}$ Department of Critical Care Medicine, Zhongshan Hospital, Fudan University, Shanghai, China; ${ }^{2}$ Center of Critical Care Medicine, The First \\ Affiliated Hospital of Xinjiang Medical University, Urumqi, China; ${ }^{3}$ Department of Anesthesiology, Zhongshan Hospital, Fudan University, \\ Shanghai, China; ${ }^{4}$ Department of Critical Care Medicine, Xiamen Branch, Zhongshan Hospital, Fudan University, Xiamen, China
}

\#These authors contributed equally to this work.

Correspondence to: Guo-Wei Tu; Zhe Luo. Department of Critical Care Medicine, Zhongshan Hospital, Fudan University, No. 180 Fenglin Road, Xuhui District, Shanghai 200032, China. Email: tu.guowei@zs-hospital.sh.cn; luo.zhe@zs-hospital.sh.cn.

Background: Fluid responsiveness is an important topic for clinicians. We investigated whether changes in left ventricular outflow tract (LVOT) velocity time integral (VTI) during a Trendelenburg position (TP) maneuver can predict fluid responsiveness as a non-invasive marker in coronary artery bypass graft (CABG) surgery patients in the operating room.

Methods: This prospective, single-center observational study, performed in the operating room, enrolled 65 elective CABG patients. Hemodynamic data coupled with transesophageal echocardiography monitoring of the LVOT VTI and the peak velocity were collected at each step [baseline 1, TP, baseline 2 and fluid challenge (FC)]. Patients whose VTI increased $\geq 15 \%$ after FC (500 mL of Gelofusine infusion within $30 \mathrm{~min}$ ) were considered responders.

Results: Twenty-eight (43.1\%) patients were responders to fluid administration. VTI changes during the TP maneuver predicted fluid responsiveness with an area under the receiver operating characteristic curve (AUC) of 0.90 (95\% CI, 0.79-0.96), with a sensitivity of $100 \%$, and a specificity of $70 \%$ at a threshold of $10 \%$ (gray zone, $8-15 \%$ ). The increase in VTI during the TP was correlated with the VTI changes induced by $\mathrm{FC}(\mathrm{r}=0.61, \mathrm{P}<0.0001)$. Changes in peak velocity and pulse pressure during the TP were poorly predictive of fluid responsiveness, with an AUC of 0.72 (95\% CI: 0.60-0.82) and 0.66 (95\% CI: 0.53-0.77), respectively. Conclusions: An increase in VTI induced by the TP could predict fluid responsiveness in CABG patients in the operating room. However, changes in peak velocity and pulse pressure stimulated by the TP could not reliably predict fluid responsiveness.

Keywords: Fluid responsiveness; transesophageal echocardiography (TEE); velocity time integral (VTI); Trendelenburg position (TP); hemodynamic monitoring; cardiac surgery

Submitted May 26, 2020. Accepted for publication Mar 03, 2021.

doi: 10.21037/qims-20-700

View this article at: http://dx.doi.org/10.21037/qims-20-700

\section{Introduction}

Hypotension is one of the most frequently encountered hemodynamic disturbances in the perioperative setting.
Treatment for hypotension typically involves intravenous fluid administration. However, in patients with impaired ventricular contractility, further volume loading is not 
accompanied by an increase in cardiac output (CO) but may lead to deterioration of cardiopulmonary function $(1,2)$. To prevent such complications, several methods have been developed to test preload responsiveness $(3,4)$. For example, passive leg raising (PLR) is a test that predicts whether $\mathrm{CO}$ will increase with volume expansion. By a gravitational shift in blood from the lower body toward the right heart, PLR mimics a fluid challenge (FC) (5). This test has been proposed as an attractive way to predict fluid responsiveness in various clinical scenarios, thus avoiding the potential harm caused by fluid loading $(6,7)$. However, PLR is not possible in the operating room. The Trendelenburg position (TP) involves placing the patient with head down and feet elevated. It is often used to treat hemodynamically unstable patients when hypovolemia is suspected (8). Having a mechanism similar to PLR, TP likewise increases venous return to the right side of the heart increasing CO (9). Patients with coronary artery bypass graft (CABG) procedures often present with a modest deterioration of global or regional ventricular contractility as well as reduced ejection fraction caused by prior myocardial ischemia (10). Therefore, perioperative fluid management should balance volume status and cardiac function. The aim of this study was to evaluate whether the TP maneuver could be used to predict fluid responsiveness in CABG patients in the operating theatre.

\section{Methods}

This was a prospective single-center observational study conducted in the cardiac surgery operating room at Zhongshan Hospital, which is affiliated with Fudan University (Shanghai, China). The study was approved by the local Ethics Committee (No. B2018-033R) and written informed consent was obtained from participating patients pre-operatively.

\section{Sample size estimation}

The sample size was estimated by using PASS software and was based on previous studies $(11,12)$. We used the following settings: $\mathrm{AUC} 0=0.5, \mathrm{AUC} 1=0.75$, power $=0.9$, alpha $=0.05$, allocation ratio $=1.0$, lower false positive rate $(\mathrm{FPR})=0$, upper $\mathrm{FPR}=1.00$, type of data $=$ continuous, and alternative hypothesis $=$ two-sided test. Therefore, the least number of measurements required was determined as 60 participants.

\section{Study population}

Patients scheduled for CABG surgery were prospectively screened. Between April and September 2019, there were 90 nonconsecutive patients who received fluid therapy in the cardiac surgical operating room. Fluid administration was performed at the discretion of the attending anesthesiologist. Criteria for fluid administration was arterial hypotension defined as a reduction of more than $20 \%$ in systolic arterial pressure with respect to the values observed before anesthesia induction. Inclusion criteria were as follows: patients older than 18 years and receiving at least 12 hours preoperative fasting. Exclusion criteria were the following: cardiac arrhythmia (e.g., atrial fibrillation or frequent premature beats), contra-indication to the TP procedure (e.g., cerebral edema or retinal detachment), contra-indication for transesophageal echocardiography (TEE) probe insertion, echocardiographic evidence of existence of moderate to severe valve disease (e.g., valve stenosis and/or regurgitation) or right heart dysfunction (right ventricular fractional area change $<35 \%$ or tricuspid annular plane systolic excursion $<16 \mathrm{~mm}$ ) (13), left ventricular ejection fraction less than $30 \%$ before surgery, intracardiac shunt, pulmonary hypertension, poor cardiac echogenicity [inability to align the Doppler beam at the left ventricular outflow tract (LVOT), and to inability to obtain a reliable measurement of velocity time integral (VTI)], lower limb amputation, or known obstruction of the inferior vena cava.

\section{Anesthetic management}

Upon arriving at the operating theatre, each patient received standard anesthetic monitoring, including five-lead electrocardiogram, pulse oximetry, and non-invasive arterial pressure measurements. Induction and maintenance of general anesthesia were performed using a target-controlled infusion of propofol TCI (target effect site concentration $2.5-3.0 \mu \mathrm{g} / \mathrm{mL})$, sufentanil $(0.3-0.5 \mu \mathrm{g} / \mathrm{kg})$ and rocuronium $(0.8 \mathrm{mg} / \mathrm{kg})$. After tracheal intubation, patients were mechanically ventilated using the volume-control mode (Primus IE, Dräger Medical, Lübeck, Germany), with a tidal volume (VT) of $8 \mathrm{~mL} / \mathrm{kg}$ predicted body weight, positive end expiratory pressure of $5 \mathrm{cmH}_{2} \mathrm{O}$, respiratory rate of 10-12 breaths per minute, inspiratory to expiratory ratio of $1: 2$, and $\mathrm{FiO}_{2}$ of $50 \%$. Ventilatory parameters were adjusted to maintain a plateau pressure less than 
$30 \mathrm{cmH}_{2} \mathrm{O}$, end-tidal carbon dioxide $\left(\mathrm{EtCO}_{2}\right)$ concentration to $35-40 \mathrm{mmHg}$ and peripheral oxygen saturation above $96 \%$. $\mathrm{EtCO}_{2}$ was continuously measured at the tip of the endotracheal tube using a sidestream infrared gas analyzer (compact airway module CAPNOTAT, Dräger, Lübeck, Germany) connected to the patient's monitor. The results were recorded online in a laptop computer every 10 seconds using proprietary data acquisition software (Dräger Medical AG \& Co. KG, Lübeck, Germany). Anesthesia was maintained with continuous infusion of propofol and sufentanil to maintain a bispectral index between 40 and 60 . Patients were excluded if the target-controlled infusion of propofol and/or sufentanil was modified during the study period.

\section{Hemodynamic monitoring}

Before induction of anesthesia, each patient received a conventional right internal jugular vein catheter (double lumen central line, ARROW, USA). After induction of anesthesia, an arterial catheter (SURFLO Flash, TERUMO, Japan) was inserted into the radial artery. The catheter was connected to a transducer and bedside Draeger monitoring. After the TEE probe was in place, the internal jugular vein catheter tip position was confirmed by the TEE in the superior vena cava. Heart rate, invasive arterial pressure and central venous pressure (CVP) were monitored continuously. The pressure transducers were positioned on the mid-axillary line and fixed to the operating table remaining at the atrial level during the TP. To minimize the effect of mechanical ventilation, CVP was measured at the end of expiration.

\section{TEE}

TEE probe insertion was routinely performed after induction of anesthesia and positioned to obtain a deep transgastric long axis view to acquire the optimum signal for velocity measurements in LVOT. This position was maintained throughout the whole study period. Echocardiographic variables were derived from the iE33 ultrasound imaging system (Philips Healthcare, Hamburg, Germany). All measurements were standardized and conducted by the same investigator (Li-Ying $\mathrm{Xu}$ ). The VTI, the peak velocity (Vmax) and the mean velocity were measured with pulsed wave Doppler through the LVOT. In order to obtain precise measurements for ultrasonic parameters, the investigator performed an average of three measurements within one TEE examination (14). For each measurement, an average of at least four consecutive cardiac beats was evaluated. If the VTI could not be measured due to anatomic reasons, or aliasing was too high because of high velocity flow, the patient was excluded from the study. Simultaneously acquired TEE images and electrocardiogram signals were recorded on a magnetooptical disk and analyzed off-line by other experienced investigators ( $\mathrm{Li}-\mathrm{Ying} \mathrm{Xu}$ and Jun-Yi Hou) blinded to the hemodynamic results.

\section{Protocol description and data collection}

This study was done after induction of anesthesia and before skin incision. Patients were studied at baseline with a $15^{\circ}$ upward bed angulation (baseline 1), during a $1 \mathrm{~min}$ postural change to the TP with a $-15^{\circ}$ downward bed angulation (TP, a shift of intravascular volume from the extra- to the intrathoracic compartment), 1 min after the end of the TP maneuver when the hemodynamic status returned to the initial baseline (baseline 2), and after a $500 \mathrm{~mL}$ of Gelofusine infusion within $30 \mathrm{~min}$ (FC) (Figure 1). Hemodynamic and TEE measurements were performed in each position. The study was terminated if the following adverse events occurred throughout the protocol: increase in heart rate $>20 \%$, decrease in peripheral oxygen saturation $<90 \%$, new onset of cardiac arrhythmia, or any other adverse event considered relevant by investigators. During the procedure, the ventilatory settings and other treatments were kept constant.

The VTI was measured from hand-drawn contours (Figure 2). The percent (\%) change in the VTI was determined by the following equation: (Post VTI - Pre VTI)/Pre VTI $\times 100 \%$. Similarly, the percent $(\%)$ change in the Vmax and pulse pressure (PP) was determined by the same equation: (Post Vmax - Pre Vmax)/Pre Vmax $\times 100 \%$ and (Post PP - Pre PP)/Pre PP $\times 100 \%$. Fluid responders were deemed present if the VTI assessed by TEE increased by at least $15 \%$ after FC, as compared to baseline 2 . This cutoff value was chosen based on previous studies (15). In addition, study participants' demographic characteristics, etiology of cardiac surgery, history of co-morbidities, preoperative cardiac function parameters, European system for cardiac operative risk evaluation, and vasoactive drug infusion doses were recorded. The recorded ventilator settings included VT, plateau pressure and $\mathrm{EtCO}_{2}$. 
A

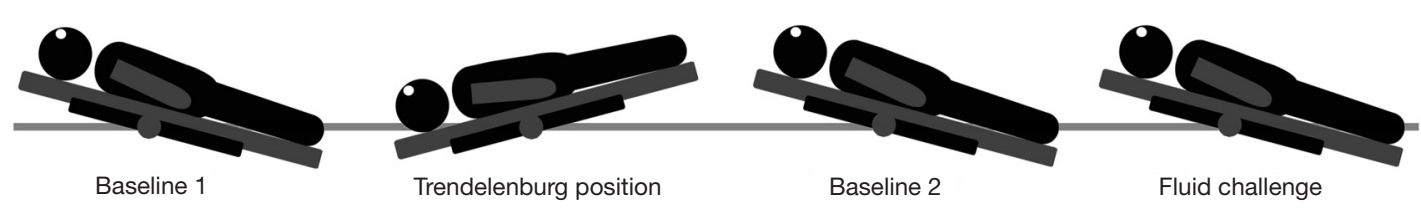

B
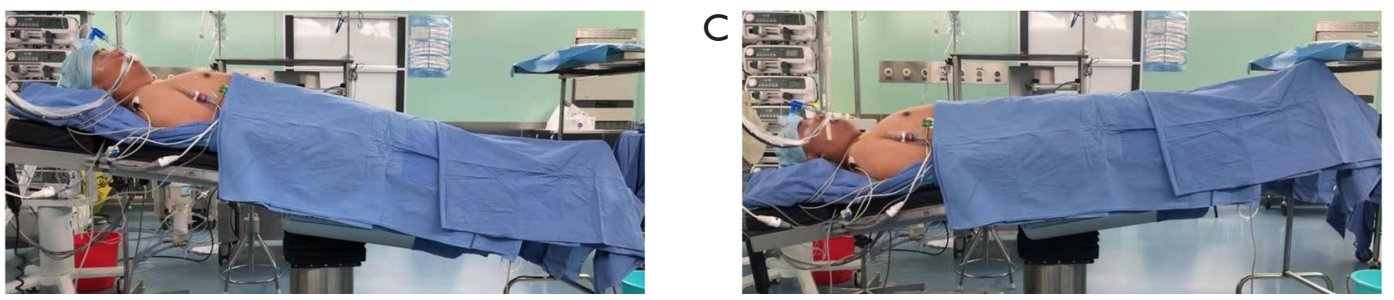

Figure 1 Study flow chart. (A) Postural change during the Trendelenburg position and design of the study procedure; (B) starting position of the Trendelenburg position with bed angulation $+15^{\circ}$; and $(\mathrm{C})$ Trendelenburg position with a $-15^{\circ}$ downward bed angulation.

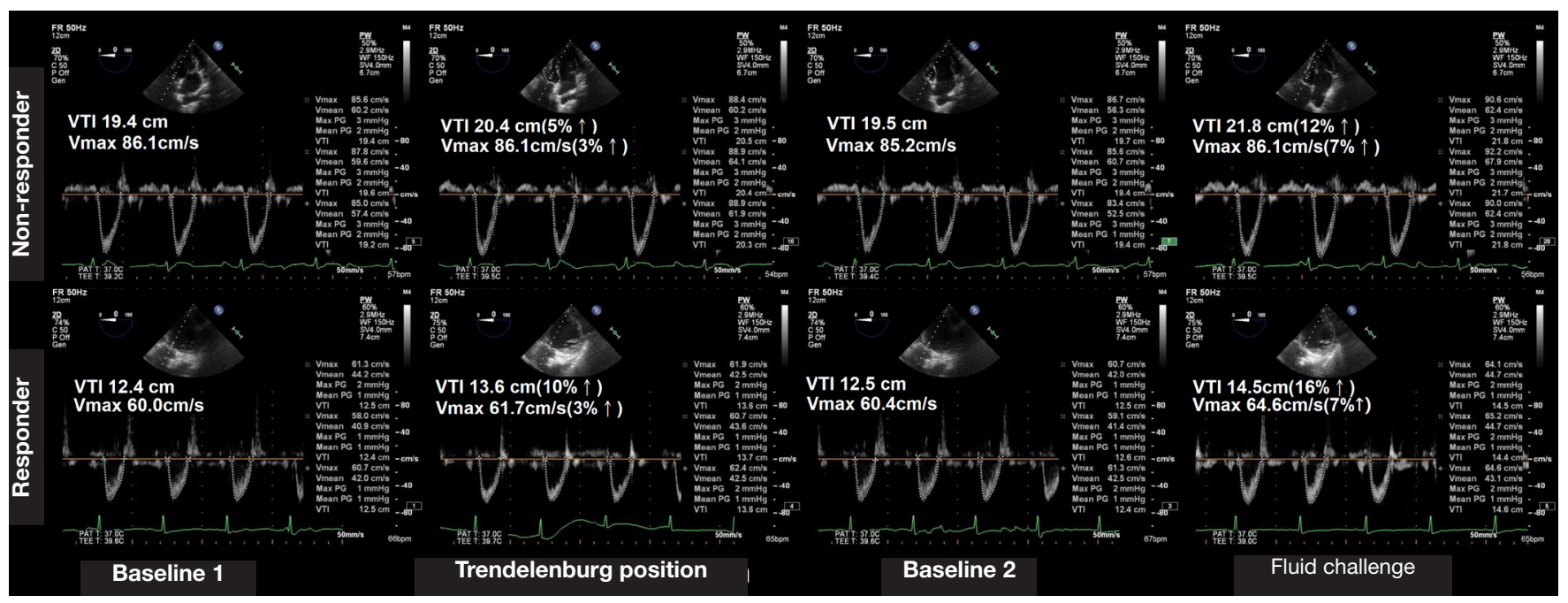

Figure 2 VTI and Vmax of the left ventricular outflow tract measured by trans-esophageal echocardiography at baselines, during Trendelenburg position, and after fluid challenge. VTI, velocity-time integral; Vmax, peak velocity.

\section{Study outcome}

The primary outcome was the diagnostic performance of LVOT VTI change during TP $[\Delta$ VTI-TP (\%)] in predicting fluid responsiveness in patients undergoing CABG surgery. The secondary outcome was diagnostic performance of the changes in Vmax and PP during TP $[\Delta \operatorname{Vmax}-\mathrm{TP}(\%)$ and $\Delta \mathrm{PP}-\mathrm{TP}(\%)]$.

\section{Statistical analysis}

The distribution of variables was assessed using the D'Agostino-Pearson test. Values are expressed as mean (standard deviation), median (25-75\% inter-quartile range) or number and proportion (\%). Comparisons within-group changes in hemodynamic variables were assessed using Student's paired $t$-test or the nonparametric Wilcoxon ranksum test, as appropriate. Differences between responders and non-responders were compared by Student's $t$-test. The relationship between $\Delta$ VTI-TP (\%) and changes in VTI induced by FC $[\Delta$ VTI-FC $(\%)]$ was tested using linear correlation. Linear correlations were tested by the Pearson rank method. A receiver-operating characteristic (ROC) curve was established for $\Delta V T I-T P, \Delta V \max -T P$, and $\Delta \mathrm{PP}-$ TP. Statistical evaluation of $\Delta$ VTI-TP, $\Delta V \max -T P$, and 


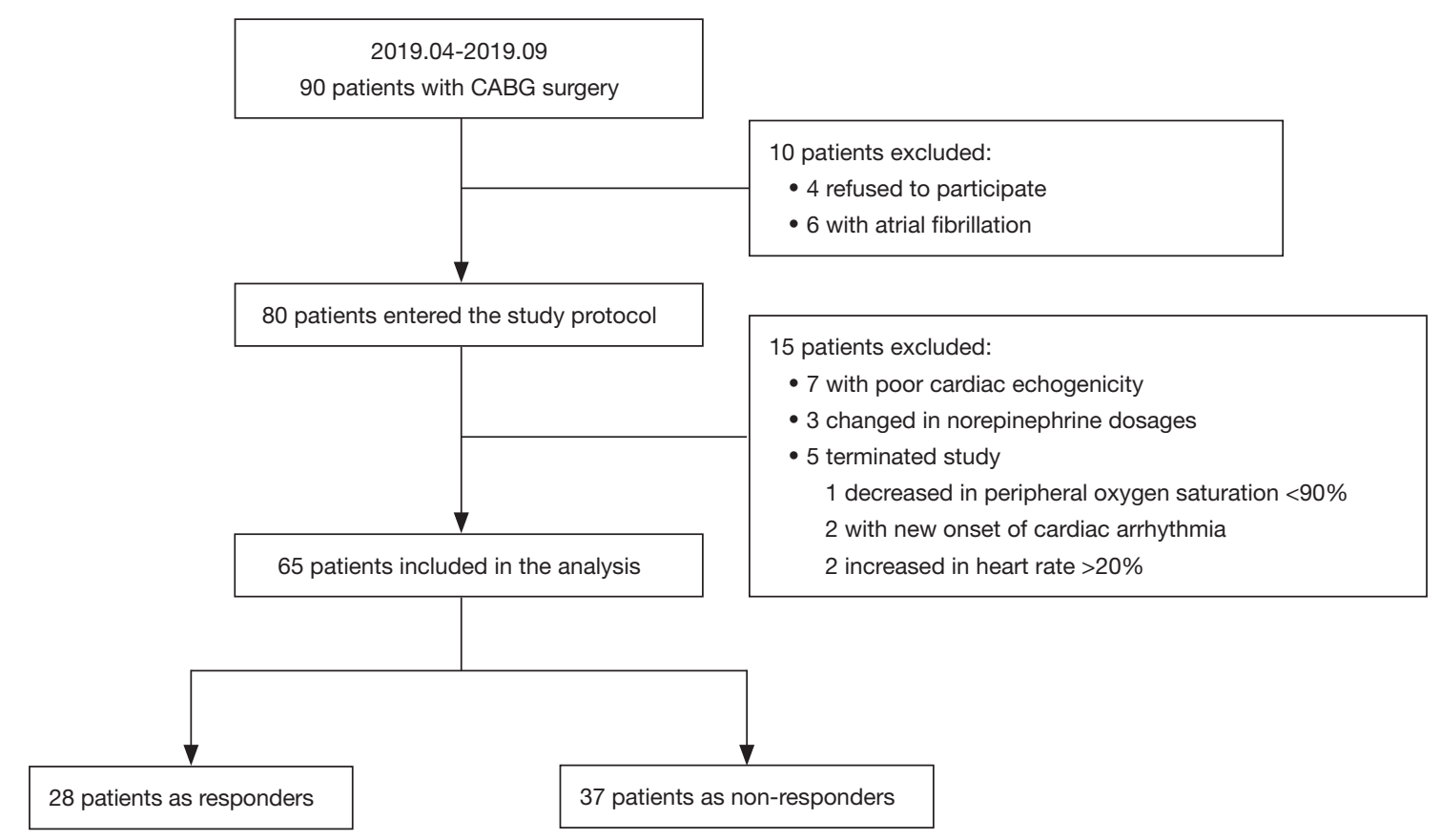

Figure 3 Flow diagram showing the patient enrollment. CABG, coronary artery bypass graft.

$\triangle \mathrm{PP}-\mathrm{TP}$ was based on areas under the ROC curve (AUC) with a $95 \%$ confidence interval (CI) and likelihood ratio. A difference between two AUCs was considered statistically significant, when the $\mathrm{P}$ value reported by DeLong et al. was $<0.05$. Statistical analysis was performed using IBM SPSS Statistics 21 (IBM, Montauk, VA, USA) and MedCalc 15.2.2 (Mariakerke, Belgium). Grey zones were constructed using a two-step procedure as described previously (16).

\section{Results}

\section{Patients' characteristics}

During the study period, 90 patients presented with inclusion criteria: four $(4.4 \%)$ patients refused to participate; six $(6.7 \%)$ were found to be in atrial fibrillation; five $(5.6 \%)$ incurred adverse events; seven $(7.8 \%)$ with poor echogenicity; and three $(3.3 \%)$ had changes in norepinephrine dosages to treat hypotension during the study period. A total of 65 patients were included in all (Figure 3). Primary characteristics, clinical complications, cardiac and respiratory parameters at inclusion are reported in Table 1. Fifty-five (84.6\%) patients received $\beta$-blockers preoperatively. Of all the patients enrolled, there were 28 (43.1\%) responders, as defined by an increase in the LVOT
VTI $\geq 15 \%$ after FC, and 37 (56.9\%) were non-responders.

\section{Hemodynamic changes after TP and after FC}

Hemodynamic and TEE data in responders and nonresponders at all study times (baseline 1, during $\mathrm{TP}$, baseline 2 and after FC) are reported in Table 2. In volume responders, TP or FC significantly decreased the heart rate. $\mathrm{TP}$ or FC induced an increase in systolic arterial pressure, mean arterial pressure, $\mathrm{PP}$, and CVP in both responders and non-responders. Responders displayed an increase in VTI and Vmax of LVOT from baseline 1 to TP or FC, but the Vmax did not change during TP nor after FC in nonresponders. In responders, VTI increased from $12 \pm 3 \mathrm{~cm}$ at baseline 2 to $15 \pm 4 \mathrm{~cm}$ after $\mathrm{FC}$. $\mathrm{EtCO}_{2}$ was higher during $\mathrm{TP}$ and after $\mathrm{FC}$ in responders, but no significant change was observed in non-responders.

\section{Changes in VTI induced by TP and by FC}

The relationship between $\Delta \mathrm{VTI}-\mathrm{TP}$ and $\Delta \mathrm{VTI}-\mathrm{FC}$ is shown in Figure $4\left(\mathrm{r}^{2}=0.37, \mathrm{P}<0.001\right)$. The relationship between these measures was closely correlated $(\mathrm{r}=0.61)$. The concordance rate between the two variables in a fourquadrant analysis with an adequate exclusion zone is shown 
Table 1 Baseline characteristics of the patients

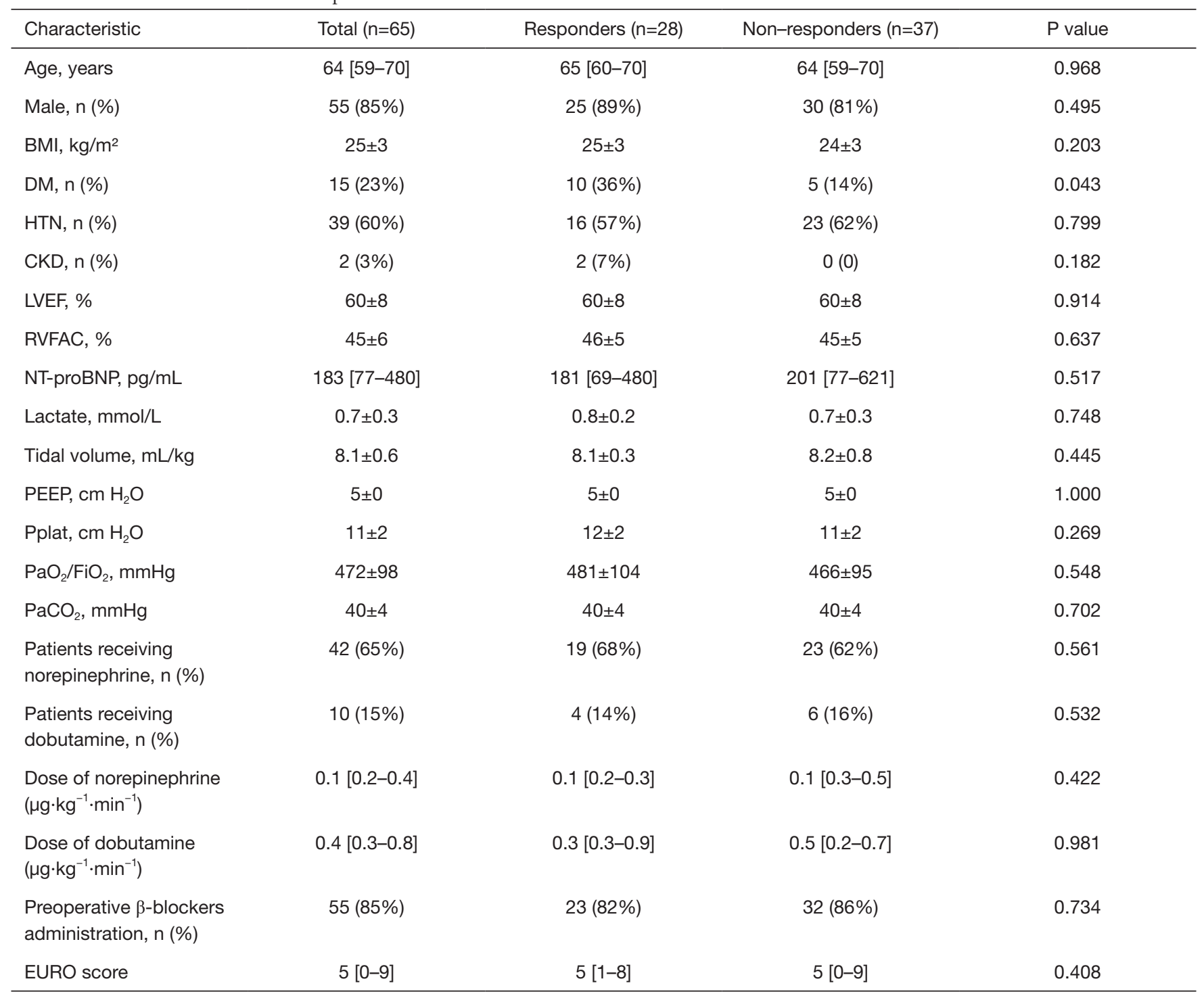

Values are expressed as the mean \pm standard deviation, median [25th-75th percentile] or number (percentage). BMI, body mass index; DM, diabetes mellitus; HTN, hypertension; CKD, chronic kidney disease; LVEF, left ventricular ejection fraction; RVFAC, right ventricular fractional area change; NT-proBNP, N-terminal pro-brain natriuretic peptide; PEEP, positive end-expiratory pressure; Pplat, airway pressure platform; $\mathrm{PaO} 2$, arterial partial pressure of oxygen; FiO2, inspiratory fraction of oxygen; $\mathrm{PaCO}$, arterial partial pressure of carbon dioxide; EURO score, European system for cardiac operative risk evaluation.

in Figure 5.

\section{Prediction of fluid responsiveness}

The prediction of $\Delta \mathrm{VTI}-\mathrm{TP}, \Delta \mathrm{Vmax}-\mathrm{TP}$ and $\Delta \mathrm{PP}-\mathrm{TP}$ to fluid responsiveness is shown in Tables 3,4 and Figure 6. The AUC generated for $\triangle$ VTI-TP $(0.90,95 \%$ CI, 0.79-0.96) was significantly higher than those generated for $\Delta \mathrm{Vmax}$ $(0.72,95 \%$ CI, $0.60-0.82)$ and $\Delta \mathrm{PP}(0.66,95 \% \mathrm{CI}, 0.53-$
0.77). The best AUC cut-off value when detecting fluid responsiveness was $>10 \%$ for $\Delta \mathrm{VTI}$, with a sensitivity of $100 \%(95 \%$ CI, $88-100 \%)$ and a specificity of $70 \%(95 \%$ CI, 53-84\%). Figure 7 shows the limits of the inconclusive zone for $\Delta \mathrm{VTI}-\mathrm{TP}, \Delta \mathrm{Vmax}-\mathrm{TP}$, and $\triangle \mathrm{PP}-\mathrm{TP}$. A large grey zone was found for $\Delta V \max$ and $\Delta \mathrm{PP}, 68 \%$ and $72 \%$ of the subjects were within this inconclusive zone, respectively. Conversely, a small grey zone for $\Delta$ VTI was observed, which included only $29 \%$ of subjects (Table 3 and Figure 7). 
Table 2 Hemodynamic parameters measured in responders and non-responders

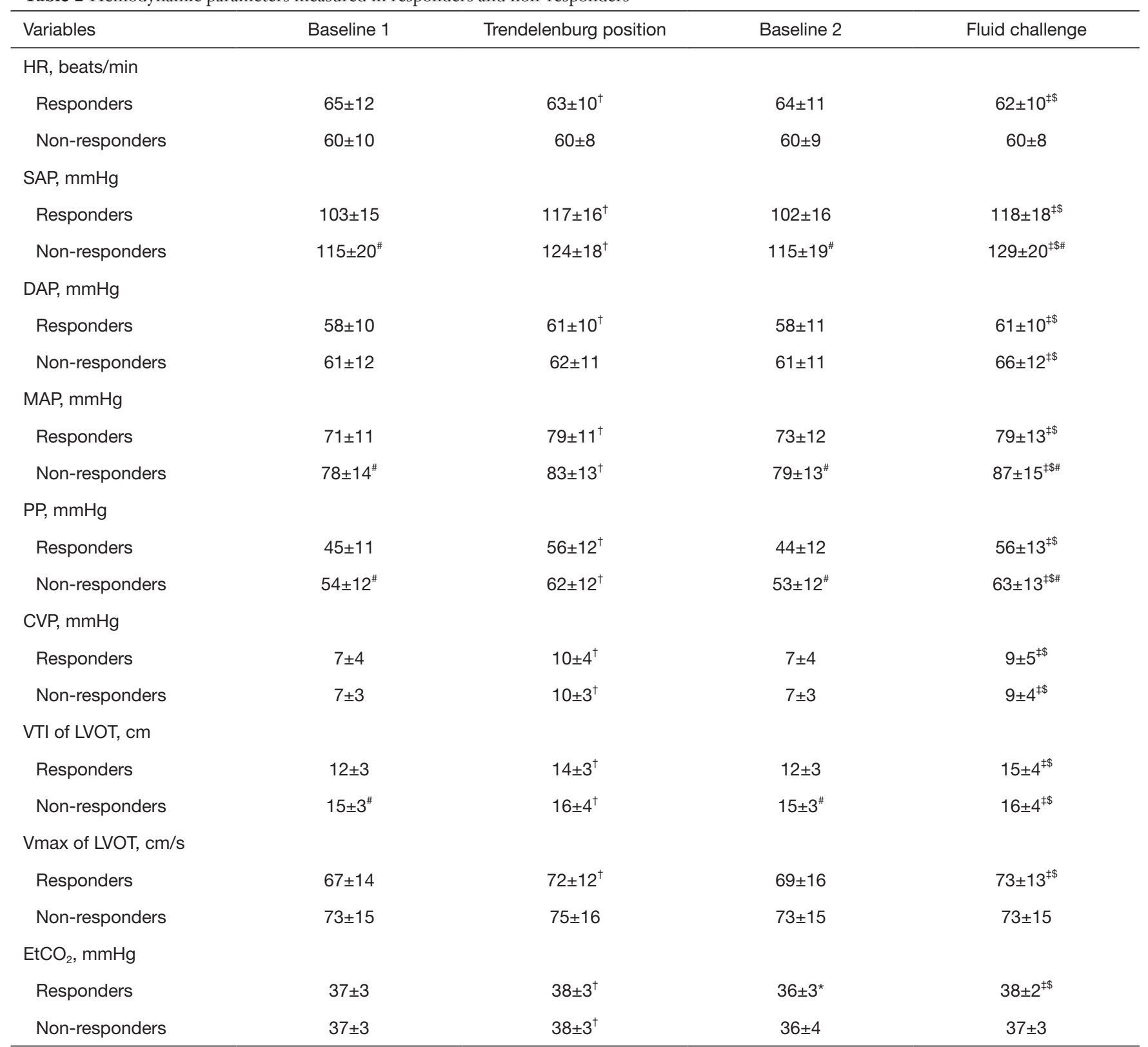

Values are expressed as mean \pm standard deviation. ${ }^{\dagger}, \mathrm{P}<0.05$ Baseline 1 vs. after Trendelenburg position; ${ }^{\ddagger}, \mathrm{P}<0.05$ Baseline 1 vs. after Fluid challenge; ${ }^{\$}, \mathrm{P}<0.05$ Baseline 2 vs. after Fluid challenge; *, $\mathrm{P}<0.05$ Baseline 1 vs. Baseline 2 ;, $\mathrm{P}<0.05$ non-responders vs. responders. HR, heart rate; SAP, systolic arterial pressure; DAP, diastolic arterial pressure; MAP, mean arterial pressure; PP, pulse pressure; CVP, central venous pressure; VTI, velocity time integral; LVOT, left ventricular outflow tract; Vmax, peak velocity; EtCO2, end-tidal carbon dioxide.

\section{Discussion}

This study demonstrated that an increase in the LVOT VTI measured by TEE induced by the TP maneuver is a highly reliable test to predict fluid responsiveness in patients undergoing coronary revascularization in the operating room.
In acute circulatory failure, PLR is a test that predicts whether $\mathrm{CO}$ will increase with volume expansion (17). However, the standardized PLR maneuver should start from a semi-recumbent position. The patient's bed must be automatically tilted to the final position with the trunk 


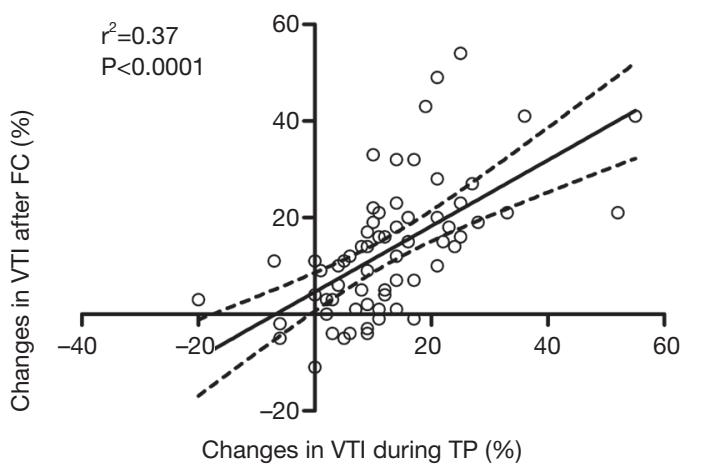

Figure 4 Relationship between percent changes in VTI during TP and after FC. VTI, velocity-time integral; TP, Trendelenburg position; FC, fluid challenge; $r^{2}$, R-squared.

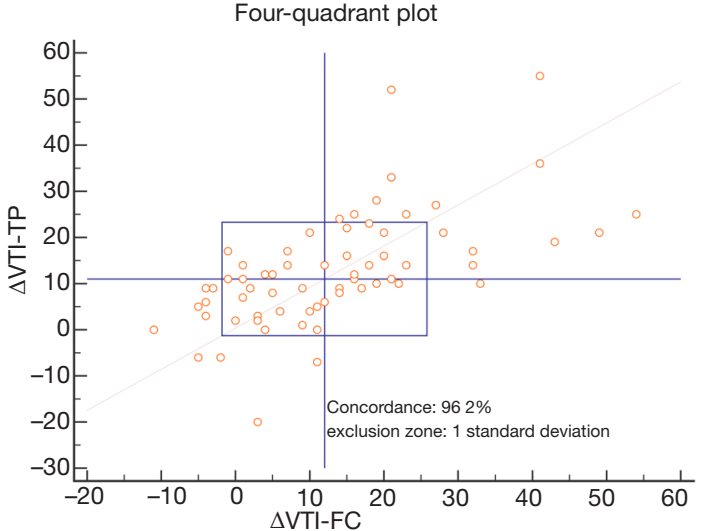

Figure 5 Four-quadrant concordance analysis between changes in VTI during TP and after FC. $\triangle$ VTI-TP, changes in velocity-time integral during the Trendelenburg position; $\triangle \mathrm{VTI}-\mathrm{FC}$, changes in velocity-time integral after fluid challenge.

Table 3 Predictive accuracy of fluid responsiveness by Trendelenburg position

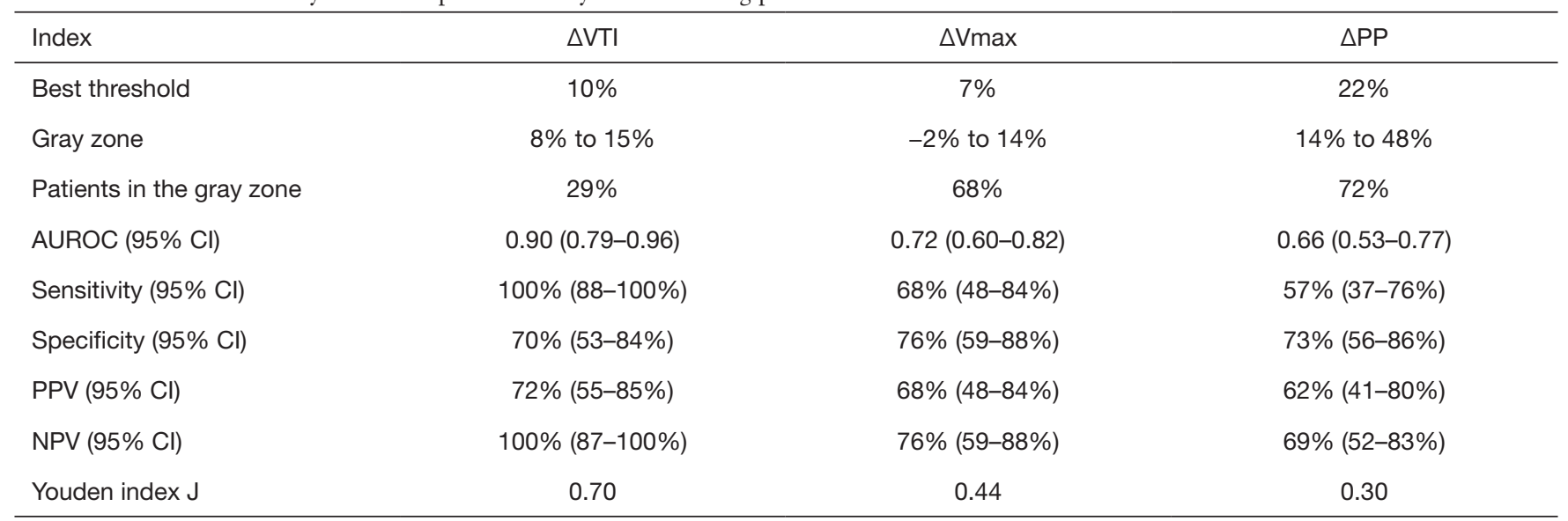

AUROC, area under the receiver operating characteristic curve; $\mathrm{Cl}$, confidence interval; $\triangle \mathrm{VTI}$, changes in VTI induced by Trendelenburg position; $\Delta V \max$, changes in peak velocity induced by Trendelenburg position; $\triangle \mathrm{PP}$, changes in pulse pressure by Trendelenburg position; PPV, positive predictive value; NPV, negative predictive value.

Table 4 The comparison of different AUROCs

\begin{tabular}{lccc}
\hline Statistic data & $\Delta$ VTI vs. $\Delta \mathrm{PP}$ & $\Delta \mathrm{PP}$ vs. $\Delta$ Vmax & \multicolumn{1}{c}{$\Delta \mathrm{VTI}$ vs. $\Delta$ Vmax } \\
\hline Differences between areas. & 0.237 & 0.062 & 0.175 \\
Standard error & 0.073 & 0.102 & 0.056 \\
$95 \% \mathrm{Cl}$ & $0.094-0.380$ & $-0.138-0.262$ & $0.065-0.286$ \\
Z statistics & 3.258 & 0.605 & 3.110 \\
P value & 0.001 & 0.545 & 0.002 \\
\hline
\end{tabular}

AUROCs, area under the receiver operating characteristic curves; $\mathrm{Cl}$, confidence interval; $\triangle \mathrm{VTI}$, changes in VTI induced by Trendelenburg position; $\triangle \mathrm{Vmax}$, changes in peak velocity induced by Trendelenburg position; $\triangle \mathrm{PP}$, changes in pulse pressure by Trendelenburg position. 
in the supine position and the legs with an elevation of $45^{\circ}$ (18). Thus, for logistical reasons, this procedure could not be implemented in the operating room. However, TP, with a mechanism similar to PLR, is commonly used to temporarily treat intraoperative suspected hypovolemia in clinical practice $(8,19)$. Rex et al. reported that the TP

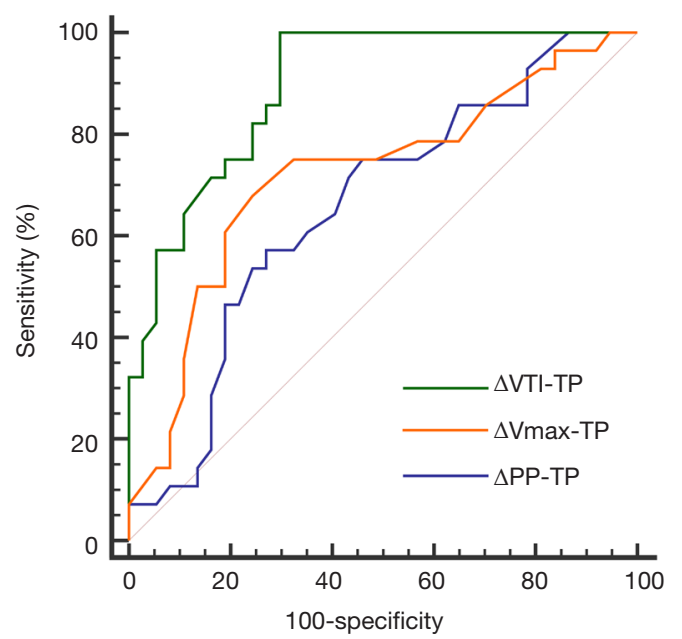

Figure 6 ROC curves generated for $\Delta$ VTI-TP, $\Delta V \max -T P$ and $\triangle \mathrm{PP}-\mathrm{TP}$ showing the ability to predict the effect of fluid challenge. Responders were defined as an increase in VTI $\geq 15 \%$ from baseline 2 after fluid challenge. ROC, receiver operating characteristics; $\Delta$ VTI-TP, changes in velocity-time integral during Trendelenburg position; $\Delta \mathrm{Vmax}-\mathrm{TP}$, changes in peak velocity during the Trendelenburg position; $\triangle \mathrm{PP}-\mathrm{TP}$, changes in pulse pressure during the Trendelenburg position. maneuver significantly increases left ventricular preload and stroke volume index, demonstrating effectiveness in inducing a relevant volume challenge (11). Likewise, Yonis et al. showed that an increase in a cardiac index $\geq 8 \%$ during TP enabled diagnosis of fluid responsiveness in acute respiratory distress syndrome patients (12). Routinely intraoperative TEE monitoring during a cardiac operation allows for correlation of functional imaging with hemodynamic measurements, determination of optimal right and left ventricular preload, guidance of inotropic therapy, and definition the effect of surgery (20). LVOT VTI was recommended as a simple, feasible and reproducible measurement to serially track CO (21). Recent studies have shown that VTI can be used for assessing the effects of some therapeutic interventions on $\mathrm{CO}$, or for performing tests assessing preload responsiveness. Lamia et al. showed that a $12.5 \%$ increase in VTI after FC with $77 \%$ sensitivity and $100 \%$ specificity can detect fluid responsiveness (22). Jozwiak et al. also demonstrated that changes in VTI $\geq 13 \%$ during consecutive end-expiratory and end-inspiratory occlusions could reliably predict fluid responsiveness (23). Thus, VTI in conjunction with the TP maneuver seems to be a feasible way to predict fluid responsiveness. In the present study, we confirmed that the reliability of $\Delta$ VTI-TP with a value of $10 \%$ in detecting fluid responsiveness, with a sensitivity of $100 \%$ and a specificity of $70 \%$. This method has the advantage of being less invasive. It might be used to test fluid responsiveness when no other technique is in place for monitoring CO. This might also be applicable and convenient in the operating theatre, especially in perioperative cardiac surgical
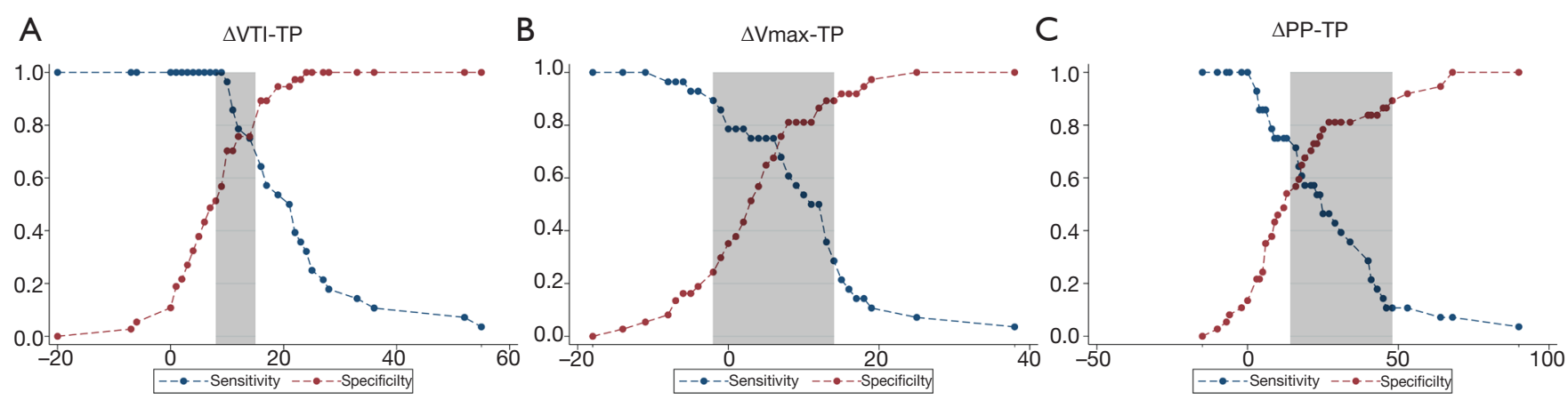

Figure 7 Two-graph ROC curves: sensitivity and specificity of changes in VTI, Vmax and PP during the TP according to the value of the cut-off for the detection of more than $15 \%$ increase in VTI after fluid challenge. The inconclusive zone, which is $>10 \%$ of diagnosis tolerance, is represented as a shaded rectangle. $\Delta$ VTI-TP, changes in velocity-time integral during the Trendelenburg position; $\Delta$ Vmax-TP, changes in peak velocity during Trendelenburg position; $\triangle \mathrm{PP}-\mathrm{TP}$, changes in pulse pressure during the Trendelenburg position. 
patients involving TEE. To the best of our knowledge, this work was the first study to evaluate the TP maneuver using TEE monitoring in predicting volume responsiveness in the operating theatre.

In the current study, $\Delta$ VTI-TP achieved a high sensitivity (100\%) and a relatively low specificity (70\%). It is speculated that TP might induce sympathetic or cardiac reflex stimulation to some extent (24). This can partially explain the relatively low specificity. $\Delta \mathrm{Vmax}-\mathrm{TP}$ was not able to predict fluid responsiveness in this study (AUC 0.72, 95\% CI: $0.60-0.82$ ). Similar to the present findings, a $\Delta \mathrm{Vmax}$ that is induced by an end-expiratory occlusion test was also found to poorly predict fluid responsiveness. This was determined in a previous study from our laboratory with an AUC of 0.75 (95\% CI: 0.63-0.86) (2). It was also found in the present study that $\triangle \mathrm{PP}-\mathrm{TP}$ could not predict fluid responsiveness (AUC 0.66, 95\% CI: 0.53-0.77). This result is consistent with our two previous studies $(2,25)$. Since PP was measured at a peripheral level and not in the aorta, we cannot exclude that the pulse wave amplification phenomenon at the peripheral level might be responsible for this result (26).

Hamzaoui et al. recently demonstrated that an increase in CVP greater than or equal to $4 \mathrm{mmHg}$ during PLR cannot identify preload unresponsiveness in critically ill patients (27). No difference in changes of CVP induced by PLR ( $\triangle$ CVP-PLR) was found between positive PLR and negative PLR test cases. There is a body of evidence indicating that a given value of CVP or change in CVP cannot predict fluid responsiveness $(28,29)$. In our study, the ability of $\triangle \mathrm{CVP}-\mathrm{TP}$ and CVP to detect fluid responsiveness was also poor, with an AUC of 0.555 (95\% CI: 0.413-0.697) and 0.511 (95\% CI: 0.369-0.653), respectively. Some studies have confirmed that variations in $\mathrm{EtCO}_{2}$ induced by PLR can track changes in the cardiac index and thus predict fluid responsiveness $(30,31)$. Toupin's study showed that an increase of $2 \mathrm{mmHg}$ in $\mathrm{EtCO}_{2}$ induced by PLR was associated with fluid responsiveness, with a sensitivity of $75 \%$ and an AUC of 0.8 (95\% CI, 0.7-0.9). Monnet et al. reported that a PLR-induced increase in $\mathrm{EtCO}_{2} \geq 5 \%$ predicted a fluid induced increase in $\mathrm{CI} \geq 15 \%$ with an AUC of 0.93 (0.810.99 ), a sensitivity of $71 \%$ and a specificity of $100 \%$. In contrast to the above findings, Arango-Granados et al. reported that variation in $\mathrm{EtCO}_{2}$ after PLR has limited utility to discriminate fluid responders among healthy spontaneously breathing adults (32). In the current study, the changes in $\mathrm{EtCO}_{2}$ induced by $\mathrm{TP}\left(\Delta \mathrm{EtCO}_{2}-\mathrm{TP}\right)$ could not predict fluid responsiveness with an AUC of 0.532 (95\% CI: 0.391-0.674).

We also acknowledge some limitations to our study. First, there was a relatively small sample of selected patients with only coronary artery disease. Whether conclusions can be extrapolated to other types of patients remains uncertain. Second, we did not use advanced hemodynamic monitoring systems (traditionally transpulmonary thermodilution devices) to measure $\mathrm{CO}$, but used VTI to track the changes in CO. Although accuracy between TEE and an advanced CO device might be questioned, there is evidence to support the use of TEE for CO-guided intraoperative fluid optimization (33-35). This technique is convenient during the operation, particularly in cardiac surgeries where intraoperative hemodynamic optimization routinely involves TEE (20). Third, ultrasound has the disadvantage of being operator-dependent. Data measured from ultrasound are better understood as semi-quantitative, because they are inherently not precise. In the current study, an average of three measurements was performed within one TEE examination. This was sufficient for obtaining precise measurements for the majority of common variables (14). In addition, TEE was performed by the same investigator and measurements were recorded blind by other investigators. The intention was to reduce intra- and inter-observer variability and echocardiographic measurement errors among clinicians dedicated to data collection. Lastly, patients with atrial fibrillation or left ventricular ejection fraction $<30 \%$ were excluded in this study. Further studies are needed to validate the conclusion in these patients.

\section{Conclusions}

This study demonstrated that an increase in VTI induced by TP could predict fluid responsiveness in CABG patients in the operating theatre. However, changes of Vmax and PP stimulated by TP could not reliably predict fluid responsiveness.

\section{Acknowledgments}

Funding: This article was supported by Research Funds of 
Shanghai Municipal Health Commission (2019ZB0105), the Natural Science Foundation of Shanghai (20ZR1411100), the Program of Shanghai Academic/Technology Research Leader (20XD1421000), the National Natural Science Foundation of China (82070085 and 82072131), Research Funds of Zhongshan Hospital (2019ZSYXQN34, 2019ZSQN13 and XYYX201922), Clinical Research Funds of Zhongshan Hospital (2020ZSLC38 and 2020ZSLC27) and Smart Medical Care of Zhongshan Hospital (2020ZHZS01).

\section{Footnote}

Conflicts of Interest: All authors have completed the ICMJE uniform disclosure form (available at http://dx.doi. org/10.21037/qims-20-700). The authors have no conflicts of interest to declare.

Ethical Statement: This study was approved by the Ethics Committee of Zhongshan Hospital affiliated with Fudan University (No. B2018-033R), and written informed consent was obtained from all study participants.

Open Access Statement: This is an Open Access article distributed in accordance with the Creative Commons Attribution-NonCommercial-NoDerivs 4.0 International License (CC BY-NC-ND 4.0), which permits the noncommercial replication and distribution of the article with the strict proviso that no changes or edits are made and the original work is properly cited (including links to both the formal publication through the relevant DOI and the license). See: https://creativecommons.org/licenses/by-nc-nd/4.0/.

\section{References}

1. Michard F, Teboul JL. Predicting fluid responsiveness in ICU patients: a critical analysis of the evidence. Chest 2002;121:2000-8.

2. Xu LY, Tu GW, Cang J, Hou JY, Yu Y, Luo Z, Guo KF. End-expiratory occlusion test predicts fluid responsiveness in cardiac surgical patients in the operating theatre. Ann Transl Med 2019;7:315.

3. Huang HB, Xu B, Liu GY, Du B. N-terminal pro-Btype natriuretic peptide for predicting fluid challenge in patients with septic shock. Ann Transl Med 2019;7:264.

4. Zhang H, Zhang Q, Chen X, Wang X, Liu D. Respiratory variations of inferior vena cava fail to predict fluid responsiveness in mechanically ventilated patients with isolated left ventricular dysfunction. Ann Intensive Care 2019;9:113.

5. Cavallaro F, Sandroni C, Marano C, La Torre G, Mannocci A, De Waure C, Bello G, Maviglia R, Antonelli $M$. Diagnostic accuracy of passive leg raising for prediction of fluid responsiveness in adults: systematic review and meta-analysis of clinical studies. Intensive Care Med 2010;36:1475-83.

6. Cherpanath TG, Hirsch A, Geerts BF, Lagrand WK, Leeflang MM, Schultz MJ, Groeneveld AB. Predicting Fluid Responsiveness by Passive Leg Raising: A Systematic Review and Meta-Analysis of 23 Clinical Trials. Crit Care Med 2016;44:981-91.

7. Mesquida J, Gruartmoner G, Ferrer R. Passive leg raising for assessment of volume responsiveness: a review. Curr Opin Crit Care 2017;23:237-43.

8. Reuter DA, Felbinger TW, Schmidt C, Moerstedt K, Kilger E, Lamm P, Goetz AE. Trendelenburg positioning after cardiac surgery: effects on intrathoracic blood volume index and cardiac performance. Eur J Anaesthesiol 2003;20:17-20.

9. Min JH, Lee SE, Lee HS, Chae YK, Lee YK, Kang Y, Je UJ. The correlation between the Trendelenburg position and the stroke volume variation. Korean J Anesthesiol 2014;67:378-83.

10. Couture P, Denault A, Limoges P, Sheridan P, Babin D, Cartier R. Mechanisms of hemodynamic changes during off-pump coronary artery bypass surgery. Can J Anaesth 2002;49:835-49.

11. Rex S, Brose S, Metzelder S, Huneke R, Schalte G, Autschbach R, Rossaint R, Buhre W. Prediction of fluid responsiveness in patients during cardiac surgery. $\mathrm{Br} \mathrm{J}$ Anaesth 2004;93:782-8.

12. Yonis H, Bitker L, Aublanc M, Perinel Ragey S, Riad Z, Lissonde F, Louf-Durier A, Debord S, Gobert F, Tapponnier R, Guerin C, Richard JC. Change in cardiac output during Trendelenburg maneuver is a reliable predictor of fluid responsiveness in patients with acute respiratory distress syndrome in the prone position under protective ventilation. Crit Care 2017;21:295.

13. Grønlykke L, Ravn HB, Gustafsson F, Hassager C, Kjaergaard J, Nilsson JC. Right ventricular dysfunction after cardiac surgery - diagnostic options. Scand 
Cardiovasc J 2017;51:114-21.

14. Jozwiak M, Mercado P, Teboul JL, Benmalek A, Gimenez J, Depret F, Richard C, Monnet X. What is the lowest change in cardiac output that transthoracic echocardiography can detect? Crit Care 2019;23:116.

15. Blanco P, Aguiar FM, Blaivas M. Rapid Ultrasound in Shock (RUSH) Velocity-Time Integral: A Proposal to Expand the RUSH Protocol. J Ultrasound Med 2015;34:1691-700.

16. Ma GG, Tu GW, Zheng JL, Zhu DM, Hao GW, Hou JY, Su Y, Luo JC, Liu K, Luo Z. Changes in Stroke Volume Variation Induced by Passive Leg Raising to Predict Fluid Responsiveness in Cardiac Surgical Patients With Protective Ventilation. J Cardiothorac Vasc Anesth 2020;34:1526-33.

17. Monnet X, Rienzo M, Osman D, Anguel N, Richard C, Pinsky MR, Teboul JL. Passive leg raising predicts fluid responsiveness in the critically ill. Crit Care Med 2006;34:1402-7.

18. Monnet X, Teboul JL. Passive leg raising: five rules, not a drop of fluid! Crit Care 2015;19:18.

19. Johnson S, Henderson SO. Myth: the Trendelenburg position improves circulation in cases of shock. CJEM 2004;6:48-9.

20. Stephens RS, Whitman GJ. Postoperative Critical Care of the Adult Cardiac Surgical Patient. Part I: Routine Postoperative Care. Crit Care Med 2015;43:1477-97.

21. Blanco P. Rationale for using the velocity-time integral and the minute distance for assessing the stroke volume and cardiac output in point-of-care settings. Ultrasound J 2020;12:21.

22. Lamia B, Ochagavia A, Monnet X, Chemla D, Richard C, Teboul JL. Echocardiographic prediction of volume responsiveness in critically ill patients with spontaneously breathing activity. Intensive Care Med 2007;33:1125-32.

23. Jozwiak M, Depret F, Teboul JL, Alphonsine JE, Lai C, Richard C, Monnet X. Predicting Fluid Responsiveness in Critically Ill Patients by Using Combined End-Expiratory and End-Inspiratory Occlusions With Echocardiography. Crit Care Med 2017;45:e1131-8.

24. London GM, Pannier B, Laurent S, Lacolley P, Safar M. Brachial artery diameter changes associated with cardiopulmonary baroreflex activation in humans. The American journal of physiology 1990;258:H773-7.

25. Hou JY, Zheng JL, Ma GG, Lin XM, Hao GW, Su Y,
Luo JC, Liu K, Luo Z, Tu GW. Evaluation of radial artery pulse pressure effects on detection of stroke volume changes after volume loading maneuvers in cardiac surgical patients. Ann Transl Med 2020;8:787.

26. De la Puente-Diaz de Leon V, de Jesus Jaramillo-Rocha V, Teboul JL, Garcia-Miranda S, Martinez-Guerra BA, Dominguez-Cherit G. Changes in Radial Artery Pulse Pressure During a Fluid Challenge Cannot Assess Fluid Responsiveness in Patients With Septic Shock. J Intensive Care Med 2020;35:149-53.

27. Hamzaoui O, Gouëzel C, Jozwiak M, Millereux M, Sztrymf B, Prat D, Jacobs F, Monnet X, Trouiller P, Teboul JL. Increase in Central Venous Pressure During Passive Leg Raising Cannot Detect Preload Unresponsiveness. Crit Care Med 2020;48:e684-9.

28. Bentzer P, Griesdale DE, Boyd J, MacLean K, Sirounis D, Ayas NT. Will This Hemodynamically Unstable Patient Respond to a Bolus of Intravenous Fluids? JAMA 2016;316:1298-309.

29. Marik PE, Cavallazzi R. Does the central venous pressure predict fluid responsiveness? An updated meta-analysis and a plea for some common sense. Crit Care Med 2013;41:1774-81.

30. Monnet X, Bataille A, Magalhaes E, Barrois J, Le Corre M, Gosset C, Guerin L, Richard C, Teboul JL. Endtidal carbon dioxide is better than arterial pressure for predicting volume responsiveness by the passive leg raising test. Intensive Care Med 2013;39:93-100.

31. Toupin F, Clairoux A, Deschamps A, Lebon JS, Lamarche Y, Lambert J, Fortier A, Denault AY. Assessment of fluid responsiveness with end-tidal carbon dioxide using a simplified passive leg raising maneuver: a prospective observational study. Can J Anaesth 2016;63:1033-41.

32. Arango-Granados MC, Zarama Córdoba V, Castro Llanos AM, Bustamante Cristancho LA. Evaluation of endtidal carbon dioxide gradient as a predictor of volume responsiveness in spontaneously breathing healthy adults. Intensive Care Med Exp 2018;6:21.

33. Monnet X, Rienzo M, Osman D, Anguel N, Richard C, Pinsky MR, Teboul JL. Esophageal Doppler monitoring predicts fluid responsiveness in critically ill ventilated patients. Intensive Care Med 2005;31:1195-201.

34. Schober P, Loer SA, Schwarte LA. Perioperative hemodynamic monitoring with transesophageal Doppler 
technology. Anesth Analg 2009;109:340-53.

35. Chang YR, Choi SH, Chang SW. Are management decisions in critical patients changed with use of

Cite this article as: Ma GG, Xu LY, Luo JC, Hou JY, Hao GW, Su Y, Liu K, Yu SJ, Tu GW, Luo Z. Change in left ventricular velocity time integral during Trendelenburg maneuver predicts fluid responsiveness in cardiac surgical patients in the operating room. Quant Imaging Med Surg 2021;11(7):3133-3145. doi: 10.21037/qims-20-700 hemodynamic parameters from transpulmonary thermodilution technique? Ann Transl Med 2019;7:370. 